\title{
An analytical study of basic skills influencing the results of Egypt sitting volleyball team in Rio de Janeiro 2016 paralympic games
}

\section{"Dr/ Ayman M.Abdel bary}

\section{Introduction and research problem:-}

Yet the achievements of the Egyptians at the Olympics (Rio de Janeiro), Brazil, in 2016 that achieved at third situation and winning the bronze medal proved to the world that the sport is not limited to abilities and competence normal, but only here they are investigating the achievements and sports victories, raising the Egyptian flag high, To determine the amount of progress and the outcome is required to measure and evaluate basic skills through the stages of training, Enabling trainers to obtain sufficient information about their teams and teams compete so useful to them in the leadership team in sports competitions and tournaments they are waging.

So it researcher sees that by using the method of analysis can access the most accurate performance skills Details of the players of the national team sitting volleyball and teams competing in the games in question, leading to the identification of the strengths and try to improve them and to identify the weaknesses and work to remedy them.

\section{Research objectives:}

1- identify the basic skills performance ratio of the Egyptian players sitting volleyball team for Total matches under discussion.

2- Identify at the level of the relative importance of the basic skills of the players, the Egyptian sitting volleyball team.

3- compare the results of games to win and defeat the basic skills of the Egyptian sitting volleyball team.

\section{Research questions:}

1- What are the performance percentage of basic skills in Egyption sitting volleyball team for Total matches under investigation?

"Lecturer, Games Department of physical Education College, Sadat City University, Egypt 
2- What is the level of the relative importance of the basic skills for the Egyptian sitting volleyball team?

3- What are the differences in the basic skills of individual results to win matches and the defeat of the Egyptian sitting volleyball team?

Research procedures:

First, the

research methodology:

The researcher used the descriptive method due to the its suitability of the nature of this study.

Second: The research sample:

The research sample was selected deliberate way, It is all the matches of the Egyptian national team winning the bronze medal in Rio de Janeiro 2016 paralympic games and the number it (5) matches.

Thirdly:tools and means of data collection:-

The researcher used through the application of research procedures scanning method for specialized scientific references and sources was used the following methods and tools to collect research data.

\section{1- Personal interview:}

The researcher designed experts questionnaires saw, in the field of volleyball and the number (3) experts Attachment (1)

\section{:2- Data registration forms}

. - Data registration form to the skill of the serve, serve reception

- - Data registration form to the skill of the set, attack hit

.- Data registration form to

skill of the blocking, defense

Fourthly: reconnaissance study:

The study was carried on two games it (USA $\times$ Germany) (China $\times$ USA) in order to identify:

- - Adequate data registration form to search skills

. - The validity of the tools and equipment used

Fifth: Basic study:

The researcher conducting the study on the number (5) matches are All matches for Egyptian national sitting volleyball team in Rio de Janeiro 2016 paralympic games, the researcher using repetitive marks in the process of analyzing the matches for the Egyptian team in these matches.

Presentation and discussion of the results: - 
Table (1)

Repetition and percentages relative importance of the set skill for Total Competition teams in the matches under discussion

\begin{tabular}{|c|c|c|c|c|c|c|c|c|}
\hline n & & $\begin{array}{l}\text { Set Type } \\
\text { ossibilities }\end{array}$ & & $\begin{array}{c}\text { Front } \\
\text { set }\end{array}$ & $\begin{array}{l}\begin{array}{l}\text { Over } \\
\text { head }\end{array} \\
\end{array}$ & $\begin{array}{c}\text { Back } \\
\text { set }\end{array}$ & $\begin{array}{l}\text { Under } \\
\text { hand }\end{array}$ & total \\
\hline \multirow[t]{4}{*}{1} & \multirow[t]{4}{*}{ Wrong set } & \multirow[t]{2}{*}{ Egypt } & repeat & 11 & 6 & 15 & 2 & 34 \\
\hline & & & $\%$ & 3.64 & 7.69 & 8.82 & 2.22 & 5.31 \\
\hline & & \multirow{2}{*}{$\begin{array}{c}\text { Competition } \\
\text { teams }\end{array}$} & repeat & 15 & 3 & 9 & 2 & 29 \\
\hline & & & $\%$ & 6.76 & 4.55 & 6.43 & 2.22 & 5.89 \\
\hline \multirow[t]{4}{*}{2} & \multirow{4}{*}{$\begin{array}{l}\text { Creates } \\
\text { free ball } \\
\text { competitor }\end{array}$} & \multirow[t]{2}{*}{ Egypt } & repeat & 38 & 21 & 9 & 26 & 94 \\
\hline & & & $\%$ & 12.58 & 26.92 & 5.29 & 28.89 & 14.69 \\
\hline & & \multirow{2}{*}{$\begin{array}{l}\text { Competition } \\
\text { teams }\end{array}$} & repeat & 29 & 12 & 7 & 23 & 71 \\
\hline & & & $\%$ & 13.06 & 18.18 & 5 & 35.94 & 14.43 \\
\hline \multirow[t]{4}{*}{3} & \multirow{4}{*}{$\begin{array}{l}\text { The set of } \\
1-2 \mathrm{~m} \\
\text { attacker } \\
\text { can dispose } \\
\text { of it }\end{array}$} & \multirow[t]{2}{*}{ Egypt } & repeat & 169 & 34 & 104 & 55 & 362 \\
\hline & & & $\%$ & 55.96 & 43.59 & 61.18 & 61.11 & 56.56 \\
\hline & & \multirow{2}{*}{$\begin{array}{c}\text { Competition } \\
\text { teams }\end{array}$} & repeat & 137 & 38 & 97 & 31 & 304 \\
\hline & & & $\%$ & 61.71 & 57.58 & 62.29 & 48.44 & 61.79 \\
\hline \multirow[t]{4}{*}{4} & \multirow{4}{*}{$\begin{array}{l}\text { set allows } \\
\text { scoring } \\
\text { points by } \\
\text { attack }\end{array}$} & \multirow[t]{2}{*}{ Egypt } & repeat & 84 & 17 & 42 & 7 & 150 \\
\hline & & & $\%$ & 27.82 & 21.80 & 24.71 & 7.78 & 23.44 \\
\hline & & \multirow{2}{*}{$\begin{array}{l}\text { Competition } \\
\text { teams }\end{array}$} & repeat & 41 & 13 & 27 & 8 & 88 \\
\hline & & & $\%$ & 18.47 & 19.70 & 19.29 & 12.5 & 17.89 \\
\hline & \multirow{4}{*}{$\begin{array}{l}\text { Total } \\
\text { Repetitions } \\
\text { And the } \\
\text { percentage }\end{array}$} & \multirow[t]{2}{*}{ Egypt } & repeat & 302 & 78 & 170 & 90 & 640 \\
\hline & & & $\%$ & $\begin{array}{l}47.19 \\
\end{array}$ & 12.19 & 26.56 & 14.06 & $100 \%$ \\
\hline & & \multirow{2}{*}{$\begin{array}{c}\text { Competition } \\
\text { teams }\end{array}$} & repeat & 222 & 66 & 140 & 64 & 492 \\
\hline & & & $\%$ & 45.12 & 13.42 & 28.46 & 13.01 & $100 \%$ \\
\hline & \multirow{2}{*}{$\begin{array}{l}\text { The } \\
\text { relative } \\
\text { weight }\end{array}$} & \multicolumn{2}{|l|}{ Egypt } & 628 & 140 & 170 & 157 & 1268 \\
\hline & & \multicolumn{2}{|c|}{ Competition teams } & 426 & 137 & 140 & 109 & 943 \\
\hline & \multirow{2}{*}{$\begin{array}{l}\text { The relative } \\
\text { importance }\end{array}$} & \multicolumn{2}{|c|}{ Egypt } & .32 & 59.83 & 67.26 & 58.15 & 66.04 \\
\hline & & \multicolumn{2}{|c|}{ Competition teams } & 63.96 & 69.19 & 67.14 & 56.77 & 63.89 \\
\hline
\end{tabular}

Shown in Table repeat percentages relative importance where ranged between (47.19) for the Front set, (12.19) for the overhead set of the Egyptian team as for The teams competition ranged between (45.12) for the Front set, (13.01) for the Under hand set. 
The relative weight $=$ total $(\mathrm{k} \times$ estimation).

$\mathrm{K}$ represents the number of repetition.
The relative importance $=$ relative weight $\div$ (total $\mathrm{k} \times$ highest esteem).

Table (2)

Repetition and percentages relative importance of the serve skill for Total Competition teams in the matches under discussion

\begin{tabular}{|c|c|c|c|c|c|c|c|}
\hline n & & $\begin{array}{l}\text { erve zone } \\
\text { ossibilities }\end{array}$ & & $\begin{array}{l}\text { Right } \\
\text { zone }\end{array}$ & $\begin{array}{l}\text { Center } \\
\text { zone }\end{array}$ & $\begin{array}{l}\text { Left } \\
\text { zone }\end{array}$ & total \\
\hline \multirow[t]{4}{*}{1} & \multirow{4}{*}{$\begin{array}{l}\text { Wrong } \\
\text { serve }\end{array}$} & \multirow{2}{*}{ Egypt } & repeat & 9 & 14 & 13 & 36 \\
\hline & & & $\%$ & 6.34 & 14.29 & 8.61 & 9.21 \\
\hline & & \multirow{2}{*}{$\begin{array}{c}\text { Competition } \\
\text { teams }\end{array}$} & repeat & 18 & 7 & 11 & 36 \\
\hline & & & $\%$ & 15.65 & 14 & 15.28 & 15.19 \\
\hline \multirow[t]{4}{*}{2} & \multirow{4}{*}{$\begin{array}{l}\text { provides } 3 \\
\text { attackers } \\
\text { competitor }\end{array}$} & \multirow[t]{2}{*}{ Egypt } & repeat & 44 & 21 & 41 & 106 \\
\hline & & & $\%$ & 30.99 & 21.43 & 27.15 & 27.11 \\
\hline & & \multirow{2}{*}{$\begin{array}{c}\text { Competition } \\
\text { teams }\end{array}$} & repeat & 33 & 13 & 8 & 54 \\
\hline & & & $\%$ & 28.70 & 26 & 11.11 & 22.79 \\
\hline \multirow[t]{4}{*}{3} & \multirow{4}{*}{$\begin{array}{l}\text { provides } \\
\text { double } \\
\text { attackers }\end{array}$} & \multirow[t]{2}{*}{ Egypt } & repeat & 42 & 15 & 25 & 82 \\
\hline & & & $\%$ & 29.58 & 15.31 & 16.56 & 20.97 \\
\hline & & \multirow{2}{*}{$\begin{array}{c}\text { Competition } \\
\text { teams }\end{array}$} & repeat & 26 & 13 & 27 & 66 \\
\hline & & & $\%$ & 22.61 & 26 & 37.5 & 27.85 \\
\hline \multirow[t]{4}{*}{4} & \multirow{4}{*}{$\begin{array}{l}\text { provides } \\
\text { single } \\
\text { attacker }\end{array}$} & \multirow[t]{2}{*}{ Egypt } & repeat & 17 & 20 & 32 & 69 \\
\hline & & & $\%$ & 11.97 & 20.41 & 21.19 & 17.65 \\
\hline & & \multirow{2}{*}{$\begin{array}{c}\text { Competition } \\
\text { teams }\end{array}$} & repeat & 13 & 7 & 9 & 29 \\
\hline & & & $\%$ & 11.30 & 14 & 12.5 & 12.24 \\
\hline \multirow[t]{4}{*}{5} & \multirow{4}{*}{$\begin{array}{c}\text { Creates free } \\
\text { ball }\end{array}$} & \multirow[t]{2}{*}{ Egypt } & repeat & 22 & 12 & 14 & 48 \\
\hline & & & $\%$ & 15.49 & 12.25 & 9.27 & 12.28 \\
\hline & & \multirow{2}{*}{$\begin{array}{c}\text { Competition } \\
\text { teams }\end{array}$} & repeat & 16 & 6 & 11 & 33 \\
\hline & & & $\%$ & 13.91 & 12 & 15.28 & 13.92 \\
\hline \multirow[t]{4}{*}{6} & \multirow{4}{*}{$\begin{array}{c}\text { Serve a } \\
\text { scoring } \\
\text { point } \\
\text { directly }\end{array}$} & \multirow[t]{2}{*}{ Egypt } & repeat & 8 & 16 & 26 & 50 \\
\hline & & & $\%$ & 5.63 & 16.33 & 17.22 & 12.79 \\
\hline & & \multirow{2}{*}{$\begin{array}{c}\text { Competition } \\
\text { teams }\end{array}$} & repeat & 9 & 4 & 6 & 19 \\
\hline & & & $\%$ & 7.83 & 8 & 8.33 & 8.02 \\
\hline
\end{tabular}

Assiut Journal For Sport Science Arts 
Follow Table (2)

Repetition and percentages relative importance of the serve skill for Total Competition teams in the matches under discussion

\begin{tabular}{|c|c|c|c|c|c|c|c|}
\hline n & \multicolumn{3}{|c|}{$\begin{array}{l}\text { Serve zone } \\
\text { Possibilities }\end{array}$} & $\begin{array}{l}\text { Right } \\
\text { zone }\end{array}$ & $\begin{array}{c}\text { Center } \\
\text { zone }\end{array}$ & $\begin{array}{l}\text { Left } \\
\text { zone }\end{array}$ & total \\
\hline & \multirow{4}{*}{$\begin{array}{c}\text { Total } \\
\text { Repetitions } \\
\text { And the } \\
\text { percentage }\end{array}$} & \multirow{2}{*}{ Egypt } & repeat & 142 & 98 & 151 & 391 \\
\hline & & & $\%$ & 36.32 & 25.06 & 38.62 & $100 \%$ \\
\hline & & \multirow{2}{*}{$\begin{array}{c}\text { Competition } \\
\text { teams }\end{array}$} & repeat & 115 & 50 & 72 & 237 \\
\hline & & & $\%$ & 48.52 & 21.10 & 30.38 & $100 \%$ \\
\hline & \multirow{2}{*}{$\begin{array}{c}\text { The relative } \\
\text { weight }\end{array}$} & \multicolumn{2}{|c|}{ Egypt } & 307 & 239 & 373 & 919 \\
\hline & & \multicolumn{2}{|c|}{ Competition teams } & 233 & 104 & 163 & 500 \\
\hline & \multirow{2}{*}{$\begin{array}{l}\text { The relative } \\
\text { importance }\end{array}$} & \multicolumn{2}{|c|}{ Egypt } & 43.24 & 81.29 & 49.40 & 47.01 \\
\hline & & \multicolumn{2}{|c|}{ Competition teams } & 40.52 & 69.33 & 45.28 & 42.19 \\
\hline
\end{tabular}

Shown in Table repeat percentages relative importance where ranged from (38.62) to serve from the left zone (25.06) to serve from the center zone of the Egyptian team while teams competition ranged between (48.52) to serve from the right zone, $(21: 10)$ to serve from Center zone.

Table (3)

Repetition and percentages relative importance of the serve reception skill for Total Competition teams in the matches under discussion

\begin{tabular}{|c|c|c|c|c|c|c|c|c|}
\hline n & & $\begin{array}{l}\text { eiving Type } \\
\text { ossibilities }\end{array}$ & & $\begin{array}{c}\text { two } \\
\text { players }\end{array}$ & $\begin{array}{l}\text { Three } \\
\text { players }\end{array}$ & $\begin{array}{l}\text { four } \\
\text { players }\end{array}$ & $\begin{array}{c}\text { Five } \\
\text { players }\end{array}$ & total \\
\hline \multirow[t]{4}{*}{1} & \multirow{4}{*}{$\begin{array}{l}\text { Wrong } \\
\text { receiving }\end{array}$} & \multirow[t]{2}{*}{ Egypt } & repeat & 1 & 7 & - & - & 8 \\
\hline & & & $\%$ & 11.11 & 4.93 & - & - & 5.30 \\
\hline & & \multirow{2}{*}{$\begin{array}{c}\text { Competition } \\
\text { teams }\end{array}$} & repeat & 2 & 37 & 3 & - & 42 \\
\hline & & & $\%$ & 15.39 & 12.72 & 33.33 & - & 13.21 \\
\hline \multirow[t]{4}{*}{2} & \multirow{4}{*}{$\begin{array}{l}\text { Creates } \\
\text { free ball }\end{array}$} & \multirow{2}{*}{ Egypt } & repeat & 4 & 22 & - & - & 26 \\
\hline & & & $\%$ & 44.44 & 15.49 & - & - & 17.22 \\
\hline & & \multirow{2}{*}{$\begin{array}{c}\text { Competition } \\
\text { teams }\end{array}$} & repeat & 2 & 33 & 4 & - & 39 \\
\hline & & & $\%$ & 15.39 & 11.34 & 44.44 & - & 12.26 \\
\hline \multirow[t]{4}{*}{3} & \multirow{4}{*}{$\begin{array}{l}\text { provides } \\
\text { single } \\
\text { attacker }\end{array}$} & \multirow[t]{2}{*}{ Egypt } & repeat & 1 & 22 & - & - & 23 \\
\hline & & & $\%$ & 11.11 & 15.49 & - & - & 15.23 \\
\hline & & \multirow{2}{*}{$\begin{array}{c}\text { Competition } \\
\text { teams }\end{array}$} & repeat & 2 & 58 & 1 & - & 61 \\
\hline & & & $\%$ & 15.39 & 19.93 & 11.11 & - & 19.18 \\
\hline
\end{tabular}


Follow Table (3)

Repetition and percentages relative importance of the serve reception skill for Total Competition teams in the matches under discussion

\begin{tabular}{|c|c|c|c|c|c|c|c|c|}
\hline$\overline{\bar{n}}$ & & $\begin{array}{l}\text { eiving Type } \\
\text { ossibilities }\end{array}$ & & $\begin{array}{c}\text { two } \\
\text { players }\end{array}$ & $\begin{array}{l}\text { Three } \\
\text { players }\end{array}$ & $\begin{array}{c}\text { four } \\
\text { players }\end{array}$ & $\begin{array}{c}\text { Five } \\
\text { players }\end{array}$ & total \\
\hline \multirow[t]{4}{*}{4} & \multirow{4}{*}{$\begin{array}{l}\text { provides } \\
\text { double } \\
\text { attackers }\end{array}$} & \multirow[t]{2}{*}{ Egypt } & repeat & 2 & 57 & - & - & 59 \\
\hline & & & $\%$ & 22.22 & 40.14 & - & - & 39.07 \\
\hline & & \multirow{2}{*}{$\begin{array}{c}\text { Competition } \\
\text { teams }\end{array}$} & repeat & 4 & 69 & 1 & - & 74 \\
\hline & & & $\%$ & 30.77 & 23.71 & 11.11 & - & 23.27 \\
\hline \multirow[t]{4}{*}{5} & \multirow{4}{*}{$\begin{array}{l}\text { provides } \\
\text { three } \\
\text { attackers }\end{array}$} & \multirow{2}{*}{ Egypt } & repeat & 1 & 34 & - & - & 35 \\
\hline & & & $\%$ & 11.11 & 23.94 & - & - & 23.18 \\
\hline & & \multirow{2}{*}{$\begin{array}{c}\text { Competition } \\
\text { teams }\end{array}$} & repeat & 3 & 94 & 4 & 1 & 102 \\
\hline & & & $\%$ & 23.08 & 32.30 & 44.44 & 100 & 32.08 \\
\hline & \multirow{4}{*}{\begin{tabular}{|c|} 
Total \\
Repetitions \\
And the \\
percentage
\end{tabular}} & \multirow{2}{*}{ Egypt } & repeat & 9 & 142 & - & - & 151 \\
\hline & & & $\%$ & 5.96 & 94.04 & - & - & $100 \%$ \\
\hline & & \multirow{2}{*}{$\begin{array}{c}\text { Competition } \\
\text { teams }\end{array}$} & repeat & 13 & 291 & 9 & 1 & 318 \\
\hline & & & $\%$ & 4.09 & 91.51 & 2.83 & 0.32 & $100 \%$ \\
\hline & \multirow{2}{*}{$\begin{array}{c}\text { The relative } \\
\text { weight }\end{array}$} & \multicolumn{2}{|l|}{ Egypt } & 16 & 373 & - & - & 389 \\
\hline & & \multicolumn{2}{|c|}{ Competition teams } & 30 & 732 & 25 & 4 & 791 \\
\hline & \multirow{2}{*}{$\begin{array}{l}\text { The relative } \\
\text { importance }\end{array}$} & \multicolumn{2}{|l|}{ Egypt } & 44.44 & 65.67 & - & - & 64.40 \\
\hline & & \multicolumn{2}{|c|}{ Competition teams } & 57.69 & 62.89 & 48.08 & 100 & 62.19 \\
\hline
\end{tabular}

Shown in Table repeat percentages relative importance where ranged from (94.04) for the receiving of three players, (5.96) for the receiving of two players for the

\section{Table (4)}

Repetition and percentages relative importance of the attack hit skill for Total Competition teams in the matches under discussion

\begin{tabular}{|c|c|c|c|c|c|c|c|c|c|c|c|}
\hline $\bar{n}$ & \multicolumn{3}{|c|}{$\begin{array}{l}\text { Attack zone } \\
\text { Possibitities }\end{array}$} & $\begin{array}{c}\text { From } \\
\text { position } \\
1\end{array}$ & $\begin{array}{c}\text { From } \\
\text { position } \\
2\end{array}$ & $\begin{array}{c}\text { From } \\
\text { position } \\
3\end{array}$ & $\begin{array}{c}\text { From } \\
\text { position } \\
4\end{array}$ & $\begin{array}{c}\text { From } \\
\text { position } \\
5\end{array}$ & $\begin{array}{c}\text { From } \\
\text { position } \\
6\end{array}$ & $\begin{array}{c}\text { Serve } \\
\text { hit }\end{array}$ & total \\
\hline \multirow[t]{4}{*}{1} & \multirow{4}{*}{$\begin{array}{l}\text { Wrong } \\
\text { attack }\end{array}$} & \multirow[t]{2}{*}{ Egypt } & repeat & - & 17 & 38 & 32 & - & - & 3 & 81 \\
\hline & & & $\%$ & - & 9.77 & 20.54 & 14.84 & - & - & 10.71 & 14.95 \\
\hline & & \multirow{2}{*}{$\begin{array}{c}\text { Competition } \\
\text { teams }\end{array}$} & repeat & - & 38 & 22 & 46 & - & - & 2 & 108 \\
\hline & & & $\%$ & - & 28.79 & 21.78 & 30.67 & - & - & 8.33 & 26.54 \\
\hline \multirow[t]{4}{*}{2} & \multirow{4}{*}{$\begin{array}{l}\text { provides } 3 \\
\text { attackers }\end{array}$} & \multirow[t]{2}{*}{ Egypt } & repeat & - & 57 & 21 & 33 & - & - & 2 & 113 \\
\hline & & & $\%$ & - & 32.76 & 11.35 & 21.29 & - & - & 7.14 & 20.85 \\
\hline & & \multirow{2}{*}{$\begin{array}{l}\text { Competition } \\
\text { teams }\end{array}$} & repeat & - & 26 & 20 & 31 & - & - & 1 & 78 \\
\hline & & & $\%$ & - & 19.70 & 19.80 & 20.67 & - & - & 4.17 & 19.17 \\
\hline
\end{tabular}

Assiut Journal For Sport Science Arts 
Follow Table (4)

Repetition and percentages relative importance of the attack hit skill for Total Competition teams in the matches under discussion

\begin{tabular}{|c|c|c|c|c|c|c|c|c|c|c|c|}
\hline $\bar{n}$ & \multicolumn{3}{|c|}{$\begin{array}{l}\text { Attack zone } \\
\text { Possibitities }\end{array}$} & $\begin{array}{c}\text { From } \\
\text { position } \\
1\end{array}$ & $\begin{array}{c}\text { From } \\
\text { position } \\
2\end{array}$ & $\begin{array}{c}\text { From } \\
\text { position } \\
3\end{array}$ & $\begin{array}{c}\text { From } \\
\text { position } \\
4\end{array}$ & $\begin{array}{c}\text { From } \\
\text { position } \\
5\end{array}$ & $\begin{array}{c}\text { From } \\
\text { position } \\
6\end{array}$ & $\begin{array}{c}\text { Serve } \\
\text { hit }\end{array}$ & total \\
\hline \multirow[t]{4}{*}{3} & \multirow{4}{*}{$\begin{array}{l}\text { provides } \\
\text { double } \\
\text { attackers }\end{array}$} & \multirow[t]{2}{*}{ Egypt } & repeat & - & 9 & 22 & 18 & - & - & 1 & 50 \\
\hline & & & $\%$ & - & 5.17 & 11.89 & 11.61 & - & - & 3.57 & 9.23 \\
\hline & & \multirow{2}{*}{$\begin{array}{c}\text { Competition } \\
\text { teams }\end{array}$} & repeat & - & 11 & 17 & 19 & - & - & 5 & 52 \\
\hline & & & $\%$ & - & 8.33 & 16.83 & 12.67 & - & - & 20.83 & 12.78 \\
\hline \multirow[t]{4}{*}{4} & \multirow{4}{*}{$\begin{array}{l}\text { provides } \\
\text { single } \\
\text { attacker }\end{array}$} & \multirow[t]{2}{*}{ Egypt } & repeat & - & 23 & 11 & 9 & - & - & 5 & 48 \\
\hline & & & $\%$ & - & 13.22 & 5.95 & 5.81 & - & - & 17.86 & 8.86 \\
\hline & & \multirow{2}{*}{$\begin{array}{c}\text { Competition } \\
\text { teams }\end{array}$} & repeat & - & 7 & 11 & 9 & - & - & 4 & 31 \\
\hline & & & $\%$ & - & 5.30 & 10.89 & 6 & - & - & 16.67 & 7.62 \\
\hline \multirow[t]{4}{*}{5} & \multirow{4}{*}{$\begin{array}{c}\text { Creates free } \\
\text { ball } \\
\text { competitor }\end{array}$} & \multirow[t]{2}{*}{ Egypt } & repeat & - & 27 & 32 & 2 & - & - & 6 & 85 \\
\hline & & & $\%$ & - & 15.52 & 17.30 & 1.29 & - & - & 21.43 & 15.68 \\
\hline & & \multirow{2}{*}{$\begin{array}{c}\text { Competition } \\
\text { teams }\end{array}$} & repeat & - & 19 & 13 & 8 & - & - & 7 & 47 \\
\hline & & & $\%$ & - & 14.39 & 12.87 & 5.33 & - & - & 29.17 & 11.55 \\
\hline \multirow[t]{12}{*}{6} & \multirow{4}{*}{$\begin{array}{c}\text { a scoring } \\
\text { point directly }\end{array}$} & \multirow[t]{2}{*}{ Egypt } & repeat & - & 41 & 61 & 52 & - & - & 11 & 165 \\
\hline & & & $\%$ & - & 23.56 & 32.97 & 33.55 & - & - & 39.29 & 30.44 \\
\hline & & \multirow{2}{*}{$\begin{array}{c}\text { Competition } \\
\text { teams }\end{array}$} & repeat & - & 31 & 18 & 37 & - & - & 5 & 91 \\
\hline & & & $\%$ & - & 23.49 & 17.8 & 24.67 & - & - & 20.83 & 22.36 \\
\hline & \multirow{4}{*}{$\begin{array}{c}\text { Total } \\
\text { Repetitions } \\
\text { And the } \\
\text { percentage }\end{array}$} & \multirow[t]{2}{*}{ Egypt } & repeat & - & 174 & 185 & 155 & - & - & 28 & 542 \\
\hline & & & $\%$ & - & 32.10 & 34.13 & 28.60 & - & - & 5.17 & 100 \\
\hline & & \multirow{2}{*}{$\begin{array}{l}\text { Competition } \\
\text { teams }\end{array}$} & repeat & - & 132 & 101 & 150 & - & - & 24 & 407 \\
\hline & & & $\%$ & - & 32.43 & 24.82 & 36.86 & - & - & 5.90 & 100 \\
\hline & \multirow{2}{*}{$\begin{array}{c}\text { The relative } \\
\text { weight }\end{array}$} & \multicolumn{2}{|c|}{ Egypt } & - & 457 & 531 & 364 & - & - & 98 & 1522 \\
\hline & & \multicolumn{2}{|c|}{ Competition teams } & - & 300 & 229 & 313 & - & - & 76 & 918 \\
\hline & \multirow{2}{*}{$\begin{array}{l}\text { The relative } \\
\text { importance }\end{array}$} & \multicolumn{2}{|c|}{ Egypt } & - & 52.53 & 57.41 & 46.97 & - & - & 70 & 56.16 \\
\hline & & \multicolumn{2}{|c|}{ Competition teams } & - & 45.46 & 45.35 & 41.33 & - & - & 63.33 & 45.11 \\
\hline
\end{tabular}

Shown in Table repeat

percentages relative importance where ranged from (34.13) to attack from position 3, (5.17) to Serve hit of the Egyptian team while teams competition ranged between (36.86) to attack from position 4 , (5.90) to Serve hit. 
Table (5)

Repetition and percentages relative importance of the defense skill for Total Competition teams in the matches under discussion

\begin{tabular}{|c|c|c|c|c|c|c|c|c|c|c|}
\hline $\bar{n}$ & \multicolumn{3}{|c|}{$\begin{array}{l}\text { defense zone } \\
\text { Possibilities }\end{array}$} & $\begin{array}{c}\begin{array}{c}\text { From } \\
\text { position } 1\end{array} \\
\end{array}$ & $\begin{array}{c}\begin{array}{c}\text { From } \\
\text { position } 2\end{array} \\
\end{array}$ & $\begin{array}{c}\begin{array}{c}\text { From } \\
\text { position } 3\end{array} \\
\end{array}$ & $\begin{array}{c}\text { From } \\
\text { position } 4\end{array}$ & $\begin{array}{c}\text { From } \\
\text { position } 5\end{array}$ & $\begin{array}{c}\text { From } \\
\text { position } 6\end{array}$ & total \\
\hline \multirow[t]{4}{*}{1} & \multirow{4}{*}{$\begin{array}{l}\text { Wrong } \\
\text { defense }\end{array}$} & \multirow[t]{2}{*}{ Egypt } & repeat & 42 & 18 & 21 & 16 & 39 & 51 & 187 \\
\hline & & & $\%$ & 25.46 & 29.03 & 32.31 & 25.40 & 22.94 & 30.36 & 24.93 \\
\hline & & \multirow{2}{*}{$\begin{array}{c}\text { Competition } \\
\text { teams }\end{array}$} & repeat & 93 & 15 & 27 & 18 & 42 & 67 & 262 \\
\hline & & & $\%$ & 39.91 & 31.25 & 38.03 & 28.13 & 23.73 & 32.06 & 32.67 \\
\hline \multirow[t]{4}{*}{2} & \multirow{4}{*}{$\begin{array}{l}\text { Creates } \\
\text { free ball } \\
\text { competitor }\end{array}$} & \multirow[t]{2}{*}{ Egypt } & repeat & 26 & 7 & 10 & 8 & 1 & 4 & 113 \\
\hline & & & $\%$ & 15.76 & 11.29 & 15.39 & 12.07 & 0.59 & 2.38 & 15.07 \\
\hline & & \multirow{2}{*}{$\begin{array}{l}\text { Competition } \\
\text { teams }\end{array}$} & repeat & 39 & 9 & 14 & 11 & 27 & 23 & 123 \\
\hline & & & $\%$ & 16.74 & 18.75 & 19.72 & 17.19 & 15.25 & 11.01 & 15.34 \\
\hline \multirow[t]{4}{*}{3} & \multirow{4}{*}{$\begin{array}{l}\text { Provides } \\
\text { single } \\
\text { attacker }\end{array}$} & \multirow[t]{2}{*}{ Egypt } & repeat & 33 & 15 & 11 & 9 & 48 & 31 & 147 \\
\hline & & & $\%$ & 20 & 24.19 & 16.92 & 14.29 & 28.24 & 18.45 & 19.60 \\
\hline & & \multirow{2}{*}{$\begin{array}{l}\text { Competition } \\
\text { teams }\end{array}$} & repeat & 46 & 15 & 13 & 16 & 40 & 31 & 161 \\
\hline & & & $\%$ & 19.74 & 31.25 & 18.31 & 25 & 22.60 & 14.83 & 20.08 \\
\hline \multirow[t]{4}{*}{4} & \multirow{4}{*}{$\begin{array}{l}\text { provides } \\
\text { double } \\
\text { attackers }\end{array}$} & \multirow[t]{2}{*}{ Egypt } & repeat & 49 & 16 & 18 & 21 & 58 & 62 & 224 \\
\hline & & & $\%$ & 29.70 & 25.81 & 27.69 & 33.33 & 34.12 & 36.91 & 29.87 \\
\hline & & \multirow{2}{*}{$\begin{array}{c}\text { Competition } \\
\text { teams }\end{array}$} & repeat & 43 & 6 & 8 & 12 & 46 & 69 & 184 \\
\hline & & & $\%$ & 18.46 & 12.5 & 11.27 & 18.75 & 25.99 & 33.01 & 22.94 \\
\hline \multirow[t]{12}{*}{5} & \multirow{4}{*}{$\begin{array}{l}\text { provides } 3 \\
\text { attackers }\end{array}$} & \multirow[t]{2}{*}{ Egypt } & repeat & 15 & 6 & 5 & 9 & 24 & 20 & 79 \\
\hline & & & $\%$ & 9.09 & 9.68 & 7.69 & 14.29 & 14.12 & 11.91 & 10.53 \\
\hline & & \multirow{2}{*}{$\begin{array}{l}\text { Competition } \\
\text { teams }\end{array}$} & repeat & 12 & 3 & 9 & 7 & 22 & 19 & 72 \\
\hline & & & $\%$ & 5.15 & 6.25 & 12.68 & 10.94 & 12.43 & 9.09 & 8.98 \\
\hline & \multirow{4}{*}{$\begin{array}{l}\text { Total } \\
\text { Repetitions } \\
\text { And the } \\
\text { percentage }\end{array}$} & \multirow[t]{2}{*}{ Egypt } & repeat & 165 & 62 & 65 & 63 & 170 & 168 & 750 \\
\hline & & & $\%$ & 22 & 8.27 & 8.67 & 8.40 & 22.67 & 22.40 & 100 \\
\hline & & \multirow{2}{*}{$\begin{array}{l}\text { Competition } \\
\text { teams }\end{array}$} & repeat & 233 & 48 & 71 & 64 & 177 & 209 & 802 \\
\hline & & & $\%$ & 29.05 & 5.99 & 8.85 & 7.98 & 22.07 & 26.06 & 100 \\
\hline & \multirow{2}{*}{$\begin{array}{c}\text { The relative } \\
\text { weight }\end{array}$} & \multicolumn{2}{|c|}{ Egypt } & 299 & 109 & 106 & 125 & 367 & 332 & 1395 \\
\hline & & \multicolumn{2}{|c|}{ Competition teams } & 308 & 69 & 100 & 49.60 & 333 & 368 & 1285 \\
\hline & \multirow{2}{*}{$\begin{array}{l}\text { The relative } \\
\text { importance }\end{array}$} & \multicolumn{2}{|c|}{ Egypt } & 45.30 & 43.95 & 40.77 & 107 & 53.97 & 49.41 & 46.50 \\
\hline & & Competitio & ams & 33.05 & 25 & 35.21 & 41.80 & 47.03 & 44.02 & 25 \\
\hline
\end{tabular}

Shown in Table repeat

percentages relative importance where ranged from (22.67) for the defense of position 5, (8.27) for the defense of position 2 of the Egyptian team while competition teams ranged between (29.05) for the defense of position 1, (5.99) for the defense of position 2 . 
Table (6)

Repetition and percentages relative importance of the block skill for Total Competition teams in the matches under discussion

\begin{tabular}{|c|c|c|c|c|c|c|c|c|c|c|c|c|c|c|}
\hline $\bar{n}$ & $\begin{array}{l}\text { block Type } \\
\text { Possibilities }\end{array}$ & & & $\begin{array}{c}\text { single } \\
\text { block } \\
\text { position } \\
2\end{array}$ & $\begin{array}{c}\text { single } \\
\text { block } \\
\text { position } \\
3\end{array}$ & $\begin{array}{c}\text { single } \\
\text { block } \\
\text { position } \\
4\end{array}$ & $\begin{array}{c}\text { Double } \\
\text { block } \\
\text { position } \\
2\end{array}$ & $\begin{array}{c}\text { Double } \\
\text { block } \\
\text { position } \\
3\end{array}$ & $\begin{array}{c}\text { Double } \\
\text { block } \\
\text { position } \\
4\end{array}$ & $\begin{array}{c}3 \\
\text { person } \\
\text { position } \\
2\end{array}$ & $\begin{array}{c}3 \\
\text { person } \\
\text { position } \\
3\end{array}$ & $\begin{array}{c}3 \\
\text { person } \\
\text { position } \\
4\end{array}$ & $\begin{array}{l}\text { Serve } \\
\text { block }\end{array}$ & total \\
\hline \multirow[t]{4}{*}{1} & \multirow{4}{*}{$\begin{array}{l}\text { Wrong } \\
\text { block }\end{array}$} & \multirow[t]{2}{*}{ Egypt } & repeat & 6 & 11 & 5 & 33 & 17 & 21 & 7 & 15 & 2 & 7 & 124 \\
\hline & & & $\%$ & 24 & 42.31 & 20 & 47.14 & 24.29 & 38.18 & 46.67 & 42.86 & 12.5 & 15.91 & 32.55 \\
\hline & & \multirow{2}{*}{$\begin{array}{l}\text { Competition } \\
\text { teams }\end{array}$} & repeat & 19 & 12 & 16 & 61 & 23 & 18 & 9 & 11 & 8 & 12 & 189 \\
\hline & & & $\%$ & 65.52 & 34.29 & 64 & 58.10 & 25 & 37.50 & 26.47 & 18.64 & 34.78 & 32.43 & 38.31 \\
\hline \multirow[t]{4}{*}{2} & \multirow{4}{*}{$\begin{array}{l}\text { block } \\
\text { bounce } \\
\text { him ball } \\
\text { and can set } \\
\text { for } \\
\text { attacker }\end{array}$} & \multirow{2}{*}{ Egypt } & repeat & 6 & 5 & 8 & 13 & 18 & 8 & 3 & 8 & 4 & 15 & 88 \\
\hline & & & $\%$ & 24 & 19.23 & 32 & 18.57 & 25.71 & 14.55 & 20 & 22.86 & 25 & 34.09 & 23.10 \\
\hline & & \multirow{2}{*}{$\begin{array}{l}\text { Competition } \\
\text { teams }\end{array}$} & repeat & 3 & 10 & 4 & 12 & 34 & 9 & 13 & 27 & 4 & 6 & 122 \\
\hline & & & $\%$ & 10.35 & 28.57 & 16 & 11.43 & 36.96 & 18.75 & 38.24 & 45.76 & 17.39 & 16.23 & 25.05 \\
\hline \multirow[t]{4}{*}{3} & \multirow{4}{*}{$\begin{array}{l}\text { block } \\
\text { creates a } \\
\text { free ball }\end{array}$} & \multirow[t]{2}{*}{ Egypt } & repeat & 2 & 2 & 7 & 8 & 11 & 15 & 2 & 2 & 6 & 7 & 62 \\
\hline & & & $\%$ & 8 & 7.69 & 28 & 11.43 & 15.71 & 27.27 & 13.33 & 5.71 & 37.5 & 15.91 & 16.27 \\
\hline & & \multirow{2}{*}{$\begin{array}{l}\text { Competition } \\
\text { teams }\end{array}$} & repeat & 1 & 4 & 1 & 21 & 18 & 12 & 5 & 8 & 6 & 8 & 84 \\
\hline & & & $\%$ & 3.45 & 11.43 & 4 & 20 & 19.57 & 25 & 14.71 & 13.56 & 26.09 & 21.62 & 17.25 \\
\hline \multirow[t]{12}{*}{4} & \multirow{4}{*}{$\begin{array}{l}\text { block } \\
\text { scoring } \\
\text { points } \\
\text { directly }\end{array}$} & \multirow[t]{2}{*}{ Egypt } & repeat & 11 & 8 & 5 & 16 & 24 & 11 & 3 & 10 & 4 & 15 & 107 \\
\hline & & & $\%$ & 44 & 30.77 & 20 & 22.86 & 34.29 & 20 & 20 & 28.57 & 25 & 34.09 & 28.08 \\
\hline & & \multirow{2}{*}{$\begin{array}{l}\text { Competition } \\
\text { teams }\end{array}$} & repeat & 6 & 9 & 4 & 11 & 17 & 9 & 7 & 13 & 5 & 11 & 92 \\
\hline & & & $\%$ & 20.69 & 25.71 & 16 & 10.48 & 18.48 & 18.75 & 20.59 & 22.03 & 21.74 & 29.73 & 18.89 \\
\hline & \multirow{4}{*}{$\begin{array}{l}\text { Total } \\
\text { Repetitions } \\
\text { And the } \\
\text { percentage }\end{array}$} & \multirow[t]{2}{*}{ Egypt } & repeat & 25 & 26 & 25 & 70 & 70 & 55 & 15 & 35 & 16 & 44 & 381 \\
\hline & & & $\%$ & 6.56 & 6.82 & 6.56 & 18.37 & 18.37 & 14.44 & 3.94 & 9.19 & 4.20 & 11.55 & 100 \\
\hline & & \multirow{2}{*}{$\begin{array}{l}\text { Competition } \\
\text { teams }\end{array}$} & repeat & 29 & 35 & 25 & 105 & 92 & 48 & 34 & 59 & 23 & 37 & 487 \\
\hline & & & $\%$ & 5.96 & 7.19 & 5.13 & 21.56 & 18.89 & 9.86 & 6.98 & 12.12 & 4.72 & 7.60 & 100 \\
\hline & \multirow{2}{*}{$\begin{array}{l}\text { The } \\
\text { relative } \\
\text { weight }\end{array}$} & \multicolumn{2}{|l|}{ Egypt } & 43 & 33 & 37 & 77 & 112 & 71 & 16 & 42 & 28 & 74 & 533 \\
\hline & & \multicolumn{2}{|c|}{ Competition teams } & 23 & 45 & 18 & 87 & 121 & 60 & 44 & 82 & 31 & 55 & 633 \\
\hline & \multirow{2}{*}{$\begin{array}{l}\text { The } \\
\text { relative } \\
\text { importance }\end{array}$} & \multicolumn{2}{|l|}{ Egypt } & 57.33 & 42.31 & 49.33 & 36.67 & 53.33 & 43.03 & 35.56 & 40 & 58.33 & 56.06 & 46.63 \\
\hline & & \multicolumn{2}{|c|}{ Competition teams } & 26.44 & 42.86 & 24 & 27.62 & 43.84 & 41.67 & 43.14 & 46.33 & 44.93 & 49.55 & 43.33 \\
\hline
\end{tabular}

Shown in Table repeat percentages relative importance where ranged from (18.37) to doubles block of position 2,3, (4.20) for the 3 person block of position 4 for

\section{Table (7)}

Repetition and percentages relative importance of the set skill for the team (Egypt - Bosnia) in a match defeat under discussion

\begin{tabular}{c|c|c|c|c|c|c|c|c}
\hline \hline $\mathbf{n}$ & \multicolumn{3}{|c|}{$\begin{array}{c}\text { Set Type } \\
\text { Possibilities }\end{array}$} & $\begin{array}{c}\text { Front } \\
\text { set }\end{array}$ & $\begin{array}{c}\text { Over } \\
\text { head }\end{array}$ & $\begin{array}{c}\text { Back } \\
\text { set }\end{array}$ & $\begin{array}{c}\text { Under } \\
\text { hand }\end{array}$ & total \\
\hline \hline \multirow{2}{*}{1} & \multirow{2}{*}{ Wrong set } & \multirow{2}{*}{ Egypt } & repeat & 1 & - & 1 & - & 2 \\
\cline { 3 - 9 } & & $\%$ & 1.82 & - & 6.67 & - & 2.50 \\
\cline { 3 - 9 } & & \multirow{2}{*}{ Bosnia } & repeat & 1 & - & - & - & 1 \\
\cline { 3 - 9 } & & $\%$ & 1.28 & - & - & - & 0.87 \\
\hline \multirow{2}{*}{2} & \multirow{2}{*}{$\begin{array}{c}\text { Creates free } \\
\text { ball competitor }\end{array}$} & \multirow{2}{*}{ Egypt } & repeat & 3 & - & - & 2 & 5 \\
\cline { 3 - 9 } & & $\%$ & 5.46 & - & - & 28.57 & 6.25 \\
\cline { 3 - 9 } & \multirow{2}{*}{ Bosnia } & repeat & 3 & 3 & - & 2 & 8 \\
\cline { 3 - 9 } & & $\%$ & 3.85 & 60 & - & 33.33 & 6.96 \\
\hline \hline
\end{tabular}

Assiut Journal For Sport Science Arts the Egyptian team while for the competition teams ranged between (21.56) for the doubles block of position 2 (4.72) for the 3 person block of position 4 . 
Follow Table (7)

Repetition and percentages relative importance of the set skill for the team (Egypt - Bosnia) in a match defeat under discussion

\begin{tabular}{|c|c|c|c|c|c|c|c|c|}
\hline $\mathbf{n}$ & \multicolumn{3}{|c|}{$\begin{array}{c}\text { Set Type } \\
\text { Possibilities }\end{array}$} & $\begin{array}{c}\text { Front } \\
\text { set }\end{array}$ & $\begin{array}{l}\text { Over } \\
\text { head }\end{array}$ & $\begin{array}{c}\text { Back } \\
\text { set }\end{array}$ & $\begin{array}{l}\text { Under } \\
\text { hand }\end{array}$ & total \\
\hline \multirow[t]{4}{*}{3} & \multirow{4}{*}{$\begin{array}{l}\text { The set of } 1- \\
2 \mathrm{~m} \text { attacker } \\
\text { can dispose of } \\
\text { it }\end{array}$} & \multirow[t]{2}{*}{ Egypt } & repeat & 45 & 2 & 14 & 5 & 66 \\
\hline & & & $\%$ & 81.82 & 66.67 & 93.33 & 71.43 & 82.50 \\
\hline & & \multirow[t]{2}{*}{ Bosnia } & repeat & 65 & 2 & 22 & 4 & 93 \\
\hline & & & $\%$ & 83.33 & 40 & 84.62 & 66.67 & 80.87 \\
\hline \multirow[t]{12}{*}{4} & \multirow{4}{*}{$\begin{array}{c}\text { set allows } \\
\text { scoring points } \\
\text { by attack }\end{array}$} & \multirow[t]{2}{*}{ Egypt } & repeat & 6 & 1 & - & - & 7 \\
\hline & & & $\%$ & 10.91 & 33.33 & - & - & 8.75 \\
\hline & & \multirow[t]{2}{*}{ Bosnia } & repeat & 9 & - & 4 & - & 13 \\
\hline & & & $\%$ & 11.54 & - & 15.39 & - & 11.30 \\
\hline & \multirow{4}{*}{$\begin{array}{c}\text { Total } \\
\text { Repetitions } \\
\text { And the } \\
\text { percentage }\end{array}$} & \multirow[t]{2}{*}{ Egypt } & repeat & 55 & 3 & 15 & 7 & 80 \\
\hline & & & $\%$ & 68.75 & 3.75 & 18.75 & 8.75 & 100 \\
\hline & & \multirow[t]{2}{*}{ Bosnia } & repeat & 78 & 5 & 26 & 6 & 115 \\
\hline & & & $\%$ & 67.83 & 4.35 & YY.TI & 5.22 & 100 \\
\hline & \multirow{2}{*}{$\begin{array}{c}\text { The relative } \\
\text { weight }\end{array}$} & \multicolumn{2}{|c|}{ Egypt } & 111 & 7 & 28 & 12 & 158 \\
\hline & & \multicolumn{2}{|c|}{ Bosnia } & 160 & 7 & 56 & 10 & 233 \\
\hline & \multirow{2}{*}{$\begin{array}{l}\text { The relative } \\
\text { importance }\end{array}$} & \multicolumn{2}{|c|}{ Egypt } & 67.27 & 77.78 & 62.22 & 57.14 & 65.83 \\
\hline & & \multicolumn{2}{|c|}{ Bosnia } & 68.38 & 46.67 & 71.80 & 55.56 & 67.54 \\
\hline
\end{tabular}

Shown in Table repeat

percentages

importance where ranged between (68.75) for the Front set, (3.75) for the overhead set of the Egyptian team as for The Bosnia team ranged between (67.83) for the Front set, (4.35) for the overhead set

Table (8)

Repetition and percentages relative importance of the serve skill for the team (Egypt - Bosnia) in a match defeat under discussion

\begin{tabular}{c|l|c|c|c|c|c|c}
\hline \hline $\mathbf{n}$ & \multicolumn{3}{|c}{$\begin{array}{c}\text { Serve zone } \\
\text { Possibilities }\end{array}$} & $\begin{array}{c}\text { Right } \\
\text { zone }\end{array}$ & $\begin{array}{c}\text { Center } \\
\text { zone }\end{array}$ & $\begin{array}{c}\text { Left } \\
\text { zone }\end{array}$ & total \\
\hline \hline 1 & \multirow{2}{*}{$\begin{array}{l}\text { Wrong } \\
\text { serve }\end{array}$} & \multirow{2}{*}{ Egypt } & repeat & - & 2 & 1 & 3 \\
\cline { 4 - 8 } & & $\%$ & - & 22.22 & 3.70 & 4.92 \\
\cline { 4 - 8 } & & Bosnia & repeat & 7 & - & 1 & 8 \\
\cline { 3 - 8 } & & $\%$ & 12.73 & - & 20 & 10.96 \\
\hline 2 & \multirow{2}{*}{$\begin{array}{l}\text { provides } \\
\text { attackers } \\
\text { competitor }\end{array}$} & \multirow{2}{*}{ Egypt } & repeat & 13 & 4 & 12 & 29 \\
\cline { 3 - 8 } & & Bosnia & repeat & 20 & 10 & 2 & 32 \\
\cline { 3 - 8 } & & $\%$ & 36.36 & 76.92 & 40 & 43.84 \\
\hline \hline
\end{tabular}


Table (8)

Repetition and percentages relative importance of the serve skill for the team (Egypt - Bosnia) in a match defeat under discussion

\begin{tabular}{|c|c|c|c|c|c|c|c|}
\hline$\overline{\mathbf{n}}$ & $\begin{array}{l}\text { Serve zone } \\
\text { Possibilities }\end{array}$ & & & $\begin{array}{l}\text { Right } \\
\text { zone }\end{array}$ & $\begin{array}{c}\text { Center } \\
\text { zone }\end{array}$ & $\begin{array}{l}\text { Left } \\
\text { zone }\end{array}$ & total \\
\hline \multirow[t]{4}{*}{3} & \multirow{4}{*}{$\begin{array}{l}\text { provides } \\
\text { double } \\
\text { attackers }\end{array}$} & \multirow[t]{2}{*}{ Egypt } & repeat & 8 & 1 & 3 & 12 \\
\hline & & & $\%$ & 32 & 11.11 & 11.11 & 19.67 \\
\hline & & \multirow[t]{2}{*}{ Bosnia } & repeat & 3 & 2 & - & 5 \\
\hline & & & $\%$ & 5.46 & 15.39 & - & 6.85 \\
\hline \multirow[t]{4}{*}{4} & \multirow{4}{*}{$\begin{array}{l}\text { provides } \\
\text { single } \\
\text { attacker }\end{array}$} & \multirow[t]{2}{*}{ Egypt } & repeat & 1 & 1 & 4 & 6 \\
\hline & & & $\%$ & 4 & 11.11 & 14.82 & 9.84 \\
\hline & & \multirow{2}{*}{ Bosnia } & repeat & 9 & 1 & 1 & 11 \\
\hline & & & $\%$ & 16.36 & 7.69 & 20 & 15.07 \\
\hline \multirow[t]{4}{*}{5} & \multirow{4}{*}{$\begin{array}{l}\text { Creates } \\
\text { free ball }\end{array}$} & \multirow{2}{*}{ Egypt } & repeat & 2 & - & 4 & 6 \\
\hline & & & $\%$ & 8 & - & 14.82 & 9.84 \\
\hline & & \multirow[t]{2}{*}{ Bosnia } & repeat & 9 & - & - & 9 \\
\hline & & & $\%$ & 16.36 & - & - & 12.33 \\
\hline \multirow[t]{4}{*}{6} & \multirow{4}{*}{$\begin{array}{l}\text { Serve } \\
\text { scoring } \\
\text { point } \\
\text { directly }\end{array}$} & \multirow[t]{2}{*}{ Egypt } & repeat & 1 & 1 & 3 & 5 \\
\hline & & & $\%$ & 4 & 11.11 & 11.11 & 8.20 \\
\hline & & \multirow[t]{2}{*}{ Bosnia } & repeat & 7 & - & 1 & 8 \\
\hline & & & $\%$ & 12.73 & - & 20 & 10.96 \\
\hline & \multirow{4}{*}{$\begin{array}{l}\text { Total } \\
\text { Repetitions } \\
\text { And the } \\
\text { percentage }\end{array}$} & \multirow[t]{2}{*}{ Egypt } & repeat & 25 & 9 & 27 & 61 \\
\hline & & & $\%$ & 40.98 & 14.75 & 44.26 & 100 \\
\hline & & \multirow[t]{2}{*}{ Bosnia } & repeat & 55 & 13 & 5 & 73 \\
\hline & & & $\%$ & 75.34 & 17.81 & 6.85 & 100 \\
\hline & \multirow{2}{*}{$\begin{array}{l}\text { The relative } \\
\text { weight }\end{array}$} & \multicolumn{2}{|l|}{ Egypt } & 45 & 14 & 61 & 120 \\
\hline & & \multicolumn{2}{|l|}{ Bosnia } & 124 & 17 & 10 & 151 \\
\hline & \multirow{2}{*}{$\begin{array}{l}\text { The } \\
\text { relative } \\
\text { importance }\end{array}$} & \multicolumn{2}{|l|}{ Egypt } & 36 & 31.11 & 45.19 & 39.34 \\
\hline & & \multicolumn{2}{|l|}{ Bosnia } & 45.09 & 26.15 & 40 & 41.37 \\
\hline
\end{tabular}

Shown in Table repeat percentages relative importance where ranged from (44.26) to serve from the left zone (14.75) to serve from the center zone of the Egyptian team while The Bosnia team ranged between (75.34) to serve from the right zone, (6.85) to serve from the left zone. 
Table (9)

Repetition and percentages relative importance of the serve reception skill for the team (Egypt - Bosnia) in a match defeat under discussion

\begin{tabular}{|c|c|c|c|c|c|c|c|c|}
\hline$\overline{\mathbf{n}}$ & \multicolumn{3}{|c|}{$\begin{array}{l}\text { receiving Type } \\
\text { Possibilities }\end{array}$} & $\begin{array}{c}\text { two } \\
\text { players }\end{array}$ & $\begin{array}{c}\text { Three } \\
\text { players }\end{array}$ & $\begin{array}{c}\text { four } \\
\text { players }\end{array}$ & $\begin{array}{c}\text { Five } \\
\text { players }\end{array}$ & total \\
\hline \multirow[t]{4}{*}{1} & \multirow{4}{*}{$\begin{array}{l}\text { Wrong } \\
\text { receiving }\end{array}$} & \multirow[t]{2}{*}{ Egypt } & repeat & - & 6 & - & - & 6 \\
\hline & & & $\%$ & - & 13.64 & - & - & 13.04 \\
\hline & & \multirow[t]{2}{*}{ Bosnia } & repeat & - & 1 & - & - & 1 \\
\hline & & & $\%$ & - & 1.61 & - & - & 1.61 \\
\hline \multirow[t]{4}{*}{2} & \multirow{4}{*}{$\begin{array}{l}\text { Creates } \\
\text { free ball }\end{array}$} & \multirow{2}{*}{ Egypt } & repeat & - & 12 & - & - & 12 \\
\hline & & & $\%$ & - & 27.27 & - & - & 26.09 \\
\hline & & \multirow{2}{*}{ Bosnia } & repeat & - & 3 & - & - & 3 \\
\hline & & & $\%$ & - & 4.84 & - & - & 4.84 \\
\hline \multirow[t]{4}{*}{3} & \multirow{4}{*}{$\begin{array}{l}\text { provides } \\
\text { single } \\
\text { attacker }\end{array}$} & \multirow[t]{2}{*}{ Egypt } & repeat & - & 4 & - & - & 4 \\
\hline & & & $\%$ & - & 9.09 & - & - & 8.70 \\
\hline & & \multirow[t]{2}{*}{ Bosnia } & repeat & - & 3 & - & - & 3 \\
\hline & & & $\%$ & - & 4.84 & - & - & 4.84 \\
\hline \multirow[t]{4}{*}{4} & \multirow{4}{*}{$\begin{array}{l}\text { provides } \\
\text { double } \\
\text { attackers }\end{array}$} & \multirow{2}{*}{ Egypt } & repeat & - & 14 & - & - & 14 \\
\hline & & & $\%$ & - & 31.82 & - & - & 30.44 \\
\hline & & \multirow[t]{2}{*}{ Bosnia } & repeat & - & 26 & - & - & 26 \\
\hline & & & $\%$ & - & 41.94 & - & - & 41.94 \\
\hline \multirow[t]{12}{*}{5} & \multirow{4}{*}{$\begin{array}{l}\text { provides } \\
\text { three } \\
\text { attackers }\end{array}$} & \multirow{2}{*}{ Egypt } & repeat & 2 & 8 & - & - & 10 \\
\hline & & & $\%$ & 100 & 18.18 & - & - & 21.74 \\
\hline & & \multirow[t]{2}{*}{ Bosnia } & repeat & - & 29 & - & - & 29 \\
\hline & & & $\%$ & - & 46.77 & - & - & 46.77 \\
\hline & \multirow{4}{*}{$\begin{array}{l}\text { Total } \\
\text { Repetitions } \\
\text { And the } \\
\text { percentage }\end{array}$} & \multirow[t]{2}{*}{ Egypt } & repeat & 2 & 44 & - & - & 46 \\
\hline & & & $\%$ & 4.35 & 95.65 & - & - & 100 \\
\hline & & \multirow[t]{2}{*}{ Bosnia } & repeat & - & 62 & - & - & 62 \\
\hline & & & $\%$ & - & 100 & - & - & 100 \\
\hline & \multirow{2}{*}{$\begin{array}{l}\text { The } \\
\text { relative } \\
\text { weight }\end{array}$} & \multicolumn{2}{|l|}{ Egypt } & 4 & 44 & - & - & 102 \\
\hline & & \multicolumn{2}{|l|}{ Bosnia } & - & 203 & - & - & 203 \\
\hline & \multirow{2}{*}{$\begin{array}{l}\text { The } \\
\text { relative } \\
\text { importance }\end{array}$} & \multicolumn{2}{|l|}{ Egypt } & 100 & 53.41 & - & - & 55.44 \\
\hline & & \multicolumn{2}{|l|}{ Bosnia } & - & 81.86 & - & - & 81.86 \\
\hline
\end{tabular}

Shown in Table repeat percentages relative importance where ranged from (95.65) for the receiving of three players, (4.35) for the receiving of two players for the Egyptian team while The Bosnia team Reached (100) for the receiving of three players. 
Table (10)

Repetition and percentages relative importance of the attack hit skill for the team (Egypt - Bosnia) in a match defeat under discussion

\begin{tabular}{|c|c|c|c|c|c|c|c|c|c|c|c|}
\hline $\bar{n}$ & $\begin{array}{l}\text { Serve zone } \\
\text { Possibilities }\end{array}$ & & & $\begin{array}{c}\text { From } \\
\text { position } \\
1 \\
\end{array}$ & $\begin{array}{c}\text { From } \\
\text { position } \\
2 \\
\end{array}$ & $\begin{array}{c}\text { From } \\
\text { position } \\
3 \\
\end{array}$ & $\begin{array}{c}\text { From } \\
\text { position } \\
4 \\
\end{array}$ & $\begin{array}{c}\text { From } \\
\text { position } \\
5 \\
\end{array}$ & $\begin{array}{c}\text { From } \\
\text { position } \\
6 \\
\end{array}$ & $\begin{array}{c}\text { Serve } \\
\text { hit }\end{array}$ & total \\
\hline \multirow[t]{4}{*}{1} & \multirow{4}{*}{$\begin{array}{l}\text { Wrong } \\
\text { attack }\end{array}$} & \multirow[t]{2}{*}{ Egypt } & repeat & - & 1 & 9 & 3 & - & - & - & 13 \\
\hline & & & $\%$ & - & 2.94 & 25.71 & 10.71 & - & - & - & 13.40 \\
\hline & & \multirow[t]{2}{*}{ Bosnia } & repeat & - & 6 & 4 & 5 & - & - & - & 15 \\
\hline & & & $\%$ & - & 16.67 & 15.39 & 18.52 & - & - & - & 16.85 \\
\hline \multirow[t]{4}{*}{2} & \multirow{4}{*}{$\begin{array}{l}\text { provides } 3 \\
\text { attackers }\end{array}$} & \multirow[t]{2}{*}{ Egypt } & repeat & - & 12 & 3 & 9 & - & - & - & 24 \\
\hline & & & $\%$ & - & 35.29 & 8.57 & 32.14 & - & - & - & 24.74 \\
\hline & & \multirow[t]{2}{*}{ Bosnia } & repeat & - & 5 & 2 & 7 & - & - & - & 14 \\
\hline & & & $\%$ & - & 13.89 & 7.69 & 25.93 & - & - & - & 15.73 \\
\hline \multirow[t]{4}{*}{3} & \multirow{4}{*}{$\begin{array}{l}\text { provides } \\
\text { double } \\
\text { attackers }\end{array}$} & \multirow[t]{2}{*}{ Egypt } & repeat & - & 1 & 4 & 3 & - & - & - & 8 \\
\hline & & & $\%$ & - & 2.94 & 11.43 & 10.71 & - & - & - & 8.25 \\
\hline & & \multirow[t]{2}{*}{ Bosnia } & repeat & - & 2 & 3 & 4 & - & - & - & 9 \\
\hline & & & $\%$ & - & 5.56 & 11.43 & 14.82 & - & - & - & 10.11 \\
\hline \multirow[t]{4}{*}{4} & \multirow{4}{*}{$\begin{array}{l}\text { provides } \\
\text { single } \\
\text { attacker }\end{array}$} & \multirow[t]{2}{*}{ Egypt } & repeat & - & 8 & 5 & 3 & - & - & - & 16 \\
\hline & & & $\%$ & - & 23.53 & 14.29 & 10.71 & - & - & - & 16.50 \\
\hline & & \multirow[t]{2}{*}{ Bosnia } & repeat & - & 2 & 5 & 3 & - & - & - & 10 \\
\hline & & & $\%$ & - & 5.56 & 19.23 & 11.11 & - & - & - & 11.24 \\
\hline \multirow[t]{4}{*}{5} & \multirow{4}{*}{$\begin{array}{l}\text { Creates } \\
\text { free ball to } \\
\text { competitor }\end{array}$} & \multirow[t]{2}{*}{ Egypt } & repeat & - & 8 & 9 & 5 & - & - & - & 22 \\
\hline & & & $\%$ & - & 23.53 & 25.71 & 17.86 & - & - & - & 22.68 \\
\hline & & \multirow[t]{2}{*}{ Bosnia } & repeat & - & 15 & 9 & 2 & - & - & - & 26 \\
\hline & & & $\%$ & - & 41.67 & 34.62 & 7.41 & - & - & - & 29.21 \\
\hline \multirow[t]{12}{*}{6} & \multirow{4}{*}{$\begin{array}{l}\text { a scoring } \\
\text { point } \\
\text { directly }\end{array}$} & \multirow{2}{*}{ Egypt } & repeat & - & 4 & 5 & 5 & - & - & - & 14 \\
\hline & & & $\%$ & - & 11.77 & 14.29 & 17.86 & - & - & - & 14.43 \\
\hline & & \multirow[t]{2}{*}{ Bosnia } & repeat & - & 6 & 3 & 6 & - & - & - & 15 \\
\hline & & & $\%$ & - & 16.67 & 11.54 & 22.22 & - & - & - & 16.85 \\
\hline & \multirow{4}{*}{$\begin{array}{l}\text { Total } \\
\text { Repetitions } \\
\text { And the } \\
\text { percentage }\end{array}$} & \multirow[t]{2}{*}{ Egypt } & repeat & - & 34 & 35 & 28 & - & - & - & 97 \\
\hline & & & $\%$ & - & 35.05 & 36.08 & 28.87 & - & - & - & 100 \\
\hline & & \multirow[t]{2}{*}{ Bosnia } & repeat & - & 36 & 26 & 27 & - & - & - & 89 \\
\hline & & & $\%$ & - & 40.45 & 29.21 & 30.34 & - & - & - & 100 \\
\hline & The & Egypt & & - & 90 & 87 & 69 & - & - & - & 246 \\
\hline & $\begin{array}{l}\text { relative } \\
\text { weight }\end{array}$ & Bosnia & & - & 105 & 74 & 62 & - & - & - & 241 \\
\hline & The & Egypt & & - & 52.94 & 49.71 & 49.29 & - & - & - & 50.72 \\
\hline & $\begin{array}{l}\text { relative } \\
\text { importance }\end{array}$ & Bosnia & & - & 58.33 & 56.92 & 45.93 & - & - & - & 54.16 \\
\hline
\end{tabular}

Shown in Table repeat percentages relative importance where ranged from (36.08) to attack from position 3 , (28.87) to attack from position 4 of the Egyptian team while The Bosnia team ranged between (40.45) to attack from position 2, (29.21) to attack from position 3 . 
Table (11)

Repetition and percentages relative importance of the defense skill for the team (Egypt - Bosnia) in a match defeat under discussion

\begin{tabular}{|c|c|c|c|c|c|c|c|c|c|c|}
\hline $\mathrm{n}$ & $\begin{array}{l}\text { defense zone } \\
\text { Possibilities }\end{array}$ & & & $\begin{array}{c}\text { From } \\
\text { position } \\
1\end{array}$ & $\begin{array}{c}\text { From } \\
\text { position } \\
2\end{array}$ & $\begin{array}{c}\text { From } \\
\text { position } \\
3\end{array}$ & $\begin{array}{c}\text { From } \\
\text { position } \\
4\end{array}$ & $\begin{array}{c}\text { From } \\
\text { position } \\
5\end{array}$ & $\begin{array}{c}\text { From } \\
\text { position } \\
6\end{array}$ & total \\
\hline \multirow[t]{4}{*}{1} & \multirow{4}{*}{$\begin{array}{l}\text { Wrong } \\
\text { defense }\end{array}$} & \multirow[t]{2}{*}{ Egypt } & repeat & 1 & - & - & - & 2 & - & 4 \\
\hline & & & $\%$ & 14.29 & - & - & - & 13.33 & - & 5.80 \\
\hline & & \multirow[t]{2}{*}{ Bosnia } & repeat & - & - & - & 1 & - & 1 & 2 \\
\hline & & & $\%$ & - & - & - & 12.5 & - & 9.09 & 2.63 \\
\hline \multirow[t]{4}{*}{2} & \multirow{4}{*}{$\begin{array}{l}\text { Creates } \\
\text { free ball to } \\
\text { competitor }\end{array}$} & \multirow[t]{2}{*}{ Egypt } & repeat & 3 & - & 1 & 2 & 5 & 2 & 13 \\
\hline & & & $\%$ & 21.43 & - & 16.67 & 40 & 33.33 & 8 & 18.84 \\
\hline & & \multirow[t]{2}{*}{ Bosnia } & repeat & 3 & 3 & 4 & - & 7 & 2 & 19 \\
\hline & & & $\%$ & 25 & 75 & 20 & - & 33.33 & 18.18 & 25.00 \\
\hline \multirow[t]{4}{*}{3} & \multirow{4}{*}{$\begin{array}{l}\text { provides } \\
\text { single } \\
\text { attacker }\end{array}$} & \multirow[t]{2}{*}{ Egypt } & repeat & 1 & 4 & 5 & - & - & 3 & 13 \\
\hline & & & $\%$ & 14.29 & 100 & 83.33 & - & - & 12 & 18.84 \\
\hline & & \multirow[t]{2}{*}{ Bosnia } & repeat & 3 & 1 & 7 & 3 & 8 & - & 22 \\
\hline & & & $\%$ & 25 & 25 & 35 & 37.5 & 38.1 & - & 28.95 \\
\hline \multirow[t]{4}{*}{4} & \multirow{4}{*}{$\begin{array}{l}\text { provides } \\
\text { double } \\
\text { attackers }\end{array}$} & \multirow[t]{2}{*}{ Egypt } & repeat & 4 & - & - & - & 3 & 4 & 11 \\
\hline & & & $\%$ & 28.57 & - & - & - & 20 & 16 & 15.94 \\
\hline & & \multirow[t]{2}{*}{ Bosnia } & repeat & 3 & - & 3 & 3 & 3 & 4 & 16 \\
\hline & & & $\%$ & 25 & - & 15 & 37.5 & 14.29 & 36.36 & 21.05 \\
\hline \multirow[t]{4}{*}{5} & \multirow{4}{*}{$\begin{array}{l}\text { provides } 3 \\
\text { attackers }\end{array}$} & \multirow[t]{2}{*}{ Egypt } & repeat & 4 & - & - & 3 & 5 & 16 & 28 \\
\hline & & & $\%$ & 28.57 & - & - & 60 & 33.33 & 64 & 40.58 \\
\hline & & \multirow[t]{2}{*}{ Bosnia } & repeat & 3 & - & 6 & 1 & 3 & 4 & 17 \\
\hline & & & $\%$ & 25 & - & 30 & 12.5 & 14.29 & 36.36 & 22.37 \\
\hline & \multirow{4}{*}{$\begin{array}{l}\text { Total } \\
\text { Repetitions } \\
\text { And the } \\
\text { percentage }\end{array}$} & \multirow[t]{2}{*}{ Egypt } & repeat & 14 & 4 & 6 & 5 & 15 & 25 & 69 \\
\hline & & & $\%$ & 20.29 & 5.80 & 8.70 & 7.25 & 21.74 & 36.23 & 100 \\
\hline & & \multirow[t]{2}{*}{ Bosnia } & repeat & 12 & 4 & 20 & 8 & 21 & 11 & 76 \\
\hline & & & $\%$ & 15.79 & 5.26 & 26.32 & 10.53 & 27.63 & 14.47 & 100 \\
\hline & \multirow{2}{*}{$\begin{array}{l}\text { The } \\
\text { relative } \\
\text { weight }\end{array}$} & \multicolumn{2}{|l|}{ Egypt } & 33 & 8 & 11 & 14 & 34 & 84 & 184 \\
\hline & & \multicolumn{2}{|l|}{ Bosnia } & 30 & 6 & 51 & 19 & 44 & 30 & 179 \\
\hline & \multirow{2}{*}{$\begin{array}{l}\text { The } \\
\text { relative } \\
\text { importance }\end{array}$} & \multicolumn{2}{|l|}{ Egypt } & 58.93 & 50 & 45.83 & 70 & 56.67 & 84 & 66.67 \\
\hline & & \multicolumn{2}{|l|}{ Bosnia } & 62.5 & 37.5 & 63.75 & 59.38 & 52.38 & 68.18 & 58.88 \\
\hline
\end{tabular}

Shown in Table repeat percentages relative importance where ranged from (36.23) for the defense of position 6, (5.80) for the defense of position 2 of the Egyptian team while The
Bosnia team ranged between (27.63) for the defense of position 5, (5.26) for the defense of position 2 .

Assiut Journal For Sport Science Arts 
Table (12)

Repetition and percentages relative importance of the block skill for the team (Egypt - Bosnia) in a match defeat under discussion

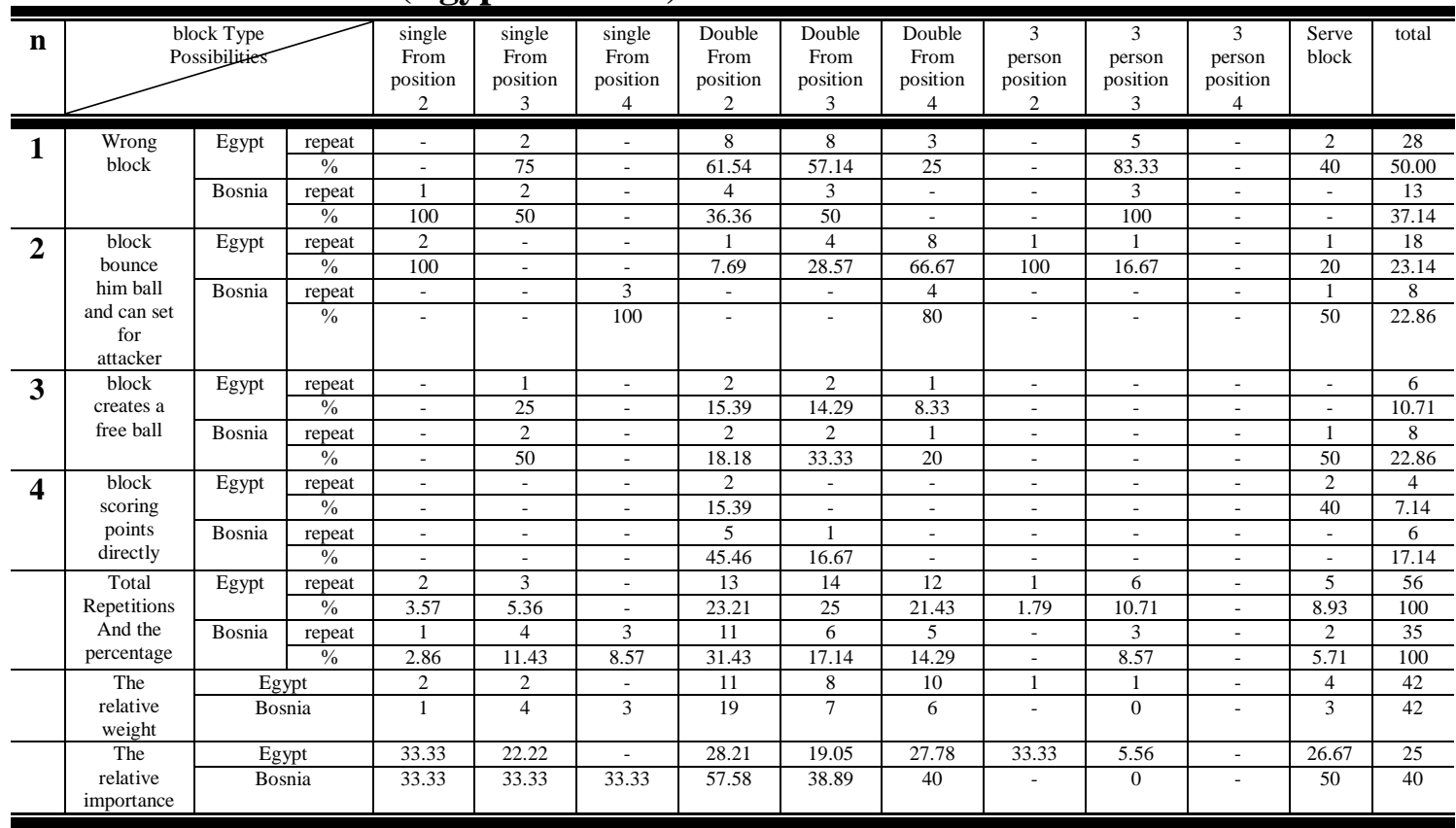

Table (13)

Repetition and percentages of basic skills under discussion

\begin{tabular}{l|l|l|l|l|l|l|l|l|l}
\hline \hline $\mathbf{n}$ & \multirow{2}{*}{ Skills } & \multicolumn{2}{|c|}{$\begin{array}{c}\text { Teams } \\
\text { defeated }\end{array}$} & \multicolumn{2}{c|}{$\begin{array}{c}\text { Egyptian } \\
\text { team }\end{array}$} & \multicolumn{2}{c|}{ Team Bosnia } & \multicolumn{2}{c}{$\begin{array}{c}\text { Total } \\
\text { matches }\end{array}$} \\
\cline { 3 - 10 } & & repeat & \% & repeat & \% & repeat & \% & repeat & \% \\
\hline \hline 1 & serve & 237 & 8.64 & 452 & 13.85 & 73 & 16.22 & 762 & 11.80 \\
\hline 2 & receiving & 318 & 11.59 & 197 & 6.04 & 62 & 13.78 & 577 & 8.94 \\
\hline 3 & defense & 802 & 29.24 & 819 & 25.09 & 76 & 16.89 & 1697 & 26.28 \\
\hline 4 & set & 492 & 17.94 & 720 & 22.06 & 115 & 25.56 & 1327 & 20.55 \\
\hline 5 & attack hit & 407 & 14.84 & 639 & 19.58 & 89 & 19.78 & 1135 & 17.58 \\
\hline 6 & block & 487 & 17.75 & 437 & 13.39 & 35 & 7.78 & 959 & 14.85 \\
\hline & Total & 2743 & 100 & 3264 & 100 & 450 & 100 & 6457 & 100 \\
\hline \hline
\end{tabular}




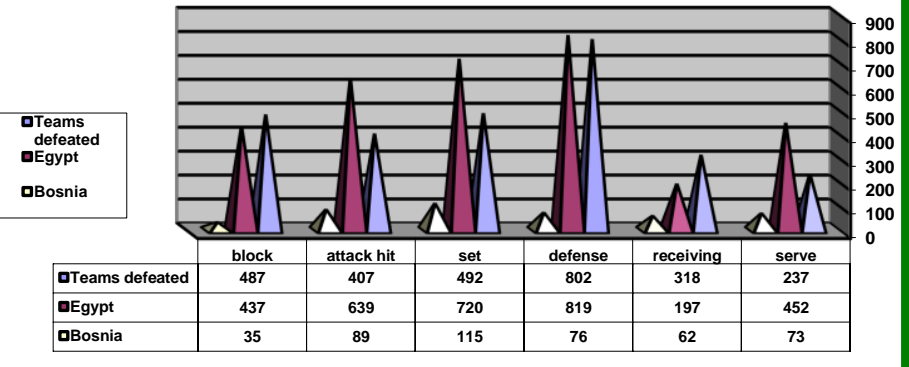

Form (1)

Repetition and percentages of basic skills

Second, discuss the results: -

Researcher discusses the findings from the reality of the data and statistical processors User in that reference previous studies framework and will attribute the researcher discuss the results of only the skill to prepare and not to the aspects of physical and tactical and psychological because it is not within the variables discussed as follows:

Discuss the results of the first question:

Shown in Table (1)The percentage has become of the largest number of repetitions for the set skill for the Egyptian team to set from 1$2 \mathrm{~m}$ attacker can attack which $56.56 \%$ and The lowest percentage of the wrong set skill $5.31 \%$, while for the

competition teams which $61.79 \%$ and the percentage of the wrong set skill $5.89 \%$.

It is clear from Table (2) the percentages for the serve skill of the Egyptian team, we have the percentage of the serve that offers three attackers $27.11 \%$, and the lowest percentage of the wrong serve $9.21 \%$, while for the competition teams of the serve that provides double attackers $27.85 \%$, and the percentage of serve scoring point directly $8.02 \%$.

Seen from the table (3) repetitions and percentages for the serve reception skill of the Egyptian team, for the receiving provides double attackers 39.07\%, and the lowest percentage of Wrong

Assiut Journal For Sport Science Arts 
receiving $5.30 \%$, while for the competition teams which the receiving provides three attackers $32.08 \%$, and the receiving creates free ball $12.26 \%$.

It is seen from the table (5) the defense skill for the Egyptian team, the percentage amounted to the largest number of repetitions to defend provides double attackers $29.87 \%$, and the lowest percentage of defense provides three attackers $10.53 \%$, while for the competition teams the percentages amounted to a larger number repetitions of the wrong defense $32.67 \%$, and the lowest percentage of defense provides three attackers $8.98 \%$. Clear from the above that wrong block for the Egyptian team representing $32.55 \%$ As for the competition teams represents $38.81 \%$ which shows that the number of repetitions for the wrong block for the Egyptian team is much less than the number of repetitions of the competition teams, which was then in the result of matches.

- Discuss the results of the second question:

It is a table (1) it is clear that the relative importance of the back set skill is the most effective for the Egyptian team and has reached (61.18) and for the competition teams was (62.29).

Seen from the table (2) that more serve zones have impact for Egyptian team the left zone was reached (38.62), But the most impact serve zones for competing teams are right zone in the matches under discussion and comes the central zone in the latter standings.

It is a table (3) it is clear that the reception of three players is more serve types impact for the Egyptian team and has reached (94.04) and for the competition teams was (91.51) in the matches under investigation.

It is clear from Table (4) the relative importance of attack hit of the front centers to be highly effective in affecting the result of the matches more than back centers, and observes that the serve hit skill for Egyptian team of little relative importance of (5.17) and for the competition teams was (5.90) the researcher return to the players serve performance of high and away from the front-row players so as not to block or serve hit allowable rules. 
The table (5) it is clear that the defense skill from the position (6) are the most effective have the Egyptian team has reached (22.40) As for total competing teams, it is the center of (1) and has reached (29.05).

\section{- Discuss the results of the third question:-}

It is clear that the set skill intermediate skill between the defensive and offensive skills and depends it largely form successful attacking hit therefore comes in first standings between the skills to win matches in the second standings matches defeat.

while the serve reception skill its come in second standings and characterized large relative importance of all the basic skills in the win matches and defeat matche.

As for The attack hit skill came in third standings between the basic skills of the win matches about the defeat matches And most influential on results Egyptian team.

It is observes that block in the fifth standings and the difficulty of movement from sitting for players who the block to try intercept the ball coming from competitors, The defense skill comes in the sixth standings and last in defeat matches and return that to the impact strength attack hits and weakness block to intercept the attack hits for competitor and the difficulty of movement for the defense well.

\section{First - conclusions: -}

Through the nature of this study and the sample and the methodology used and the results of the statistical analysis in the scope of this research the researcher suggested the following conclusions:

1- more skills to use in the matches under investigation it the defense skill, followed the set skill followed attack hit skill followed block skill.

2- The effect of basic skills in terms of relative importance in winning matches under discussion it the set skill followed the reception skill followed the attack hit skill followed the serve skill followed the block skill and the defense skill.

\section{Recommendations: -}

the researcher was suggested to identify recommendations that benefit the work in the field of training for volleyball players is as follows:

1.Training on performance the serve of the right and central zone. 
2.Training in the use of the types of attack from the set especially of position 4 and also position ( 3.2 ), and training to hit and block the serve and that as they have effective role in the results of matches.

\section{References:}

1- Abdel Ati Abdel Fattah: the effect of suggested training program for volleyball juniors on the development of some performances skills extracted from analysis of the World Cup 1995, Ph.D. thesis, unpublished, University of the Suez Canal, 1998.

\section{2- Mahmoud Wajih Hamdi:}

The impact of the low defense skill to the results of the matches in volleyball, Master Thesis, unpublished, Faculty of Physical Education-Boys, Helwan University, 1987.

\section{3- Mohamed}

Hassanein Hamdi Abdel Moneim: the scientific foundations for volleyball and measurement methods, the book publishing center, Cairo, 1997.
4- Mostafa Ahmed Sadeq:

Kinetic analysis of technical performance have volleyball players physically disabled, Ph.D. thesis, unpublished, Faculty of Physical Education, Tanta University, 1992.

\section{5- Osama El-Sherbini el} sayd: An analytical Study of basic skills influencing results of egypt sitting volleyball national team in Sydney 2000 Olympic games, Master Thesis, unpublished, Faculty of Physical Education, Tanta University, 2003.

6- World organisation volleyball for disabled: International rules for sitting volleyball, Cairo, 2012.:

\section{7- Zaki Mohammed Hassan:}

An analytical study of the level of performance skills to some volleyball teams common in Los Angeles 1984 Olympic Games, published research, Physical Education Research Journal, Faculty of Physical Education for Boys, Zagazig University, 1987. 